\title{
Über das Maximum des absoluten Betrages eines Polynoms in einem gegebenen Intervall.
}

Von

I. Schur in Berlin.

Bei seinen Untersuchungen über die Approximation stetiger Funktionen durch Polynome ist Tschebyschef bekanntlich auf eine Reihe merkwürdiger Sätze über das Verhalten des absoluten Betrages eines Polynoms in einem gegebenen Intervall geführt worden. Weitere interessante Resultate verdankt man insbesondere Herrn A. Markoff, seinem jung verstorbenen Bruder W. Markoff und in neuerer Zeit Herm S. Bernstein. Eines der schönsten und einfachsten unter diesen Resultaten ist der bekannte Satz des Ferrn A. Markoff ${ }^{1}$ ) über die Ableitung eines gegebenen Polynoms. Im folgenden will ich zeigen, daß dieser Satz sich nach verschiedenen Richtungen hin noch wesentlich ergänzen läßt. Insbesondere gebe ich an, wie der A. Markoffsche Satz zu modifizieren ist, wenn man nur solche Polynome in Betracht zieht, die an einem oder an beiden Endpunkten des vorgeschriebenen Intervalls verschwinden. In engem Zusammenhang hiermit steht die Frage nach den Beziehungen zwischen dem absoluten Betrage eines für $x=0$ verschwindenden Polynoms $f(x)$ und dem von $\frac{f(x)}{x}$. Bei diesen Untersuchungen bediene ich mich der einfachen Beweismethode, die Herr Mr. Riesz ${ }^{*}$ ) mit ausgezeichnetem Erfolg zur Herleitung und Vertiefung einiger der Hauptsätze dieser Theorie benutzt hat. Sie beruht in erster Linie auf der Heranziehung passend gewählter Interpolationsformeln.

1) "Utber ein Problem von D. J. Mendelejeff" (russisch), Abh. der Akad. der Wiss. zu St. Petersburg, Bd. 62, (1889), S. 1-24.

2), Wine trigionometrische Interpolationsformel and einige Ungleichungen für Polynome", Jahresber. der Deutschen Math.-Ver., Bd. 23 (1914), S. 354-368, und "tber einen Satz des Herrn Serge Bernstein ", Acta Math., Bd. 40 (1916), S. 337 bis 347. - Die zuerst genannte Arbeit werde ich im folgenden mit I zitieren. 
$\$ 1$.

\section{Kurze Herleitung des A. Markoffschen Satzes.}

Die Koeffizienten der im folgenden zu betrachtenden Polynome sollen, wenn nicht das Gegenteil hervorgehoben wird, beliebige komplexe Zahlen bedeuten können. Das Maximum einer (stetigen) Funktion $\varphi(x)$ in einem endlichen Intervall $a \leqq x \leqq b$ bezeichne ich mit $\operatorname{Max}(\varphi ; a, b)$. Unter $T_{n}(x)$ verstehe ich wie üblich das von Tschebyschef so oft betrachtete Polynom

$$
\begin{aligned}
T_{n}(x)=\cos (n \arccos x) & =\frac{1}{2}\left[\left(x+\sqrt{x^{2}}-1\right)^{n}+\left(x-\sqrt{x^{2}-1}\right)^{n}\right]= \\
& =\sum_{x=0}^{\left[\frac{n}{2}\right]} \frac{(-1)^{n} n}{2(n-v)}\left(\begin{array}{c}
n-v \\
y
\end{array}\right)(2 x)^{n-2,} .
\end{aligned}
$$

Es wird also

$$
\begin{gathered}
T_{n}(\cos \varphi)=\cos n \varphi, \quad T_{n}^{\prime}(\cos \varphi)=\frac{n \sin n \varphi}{\sin \varphi}, \\
\operatorname{Max}\left(\left|T_{n}\right| ;-1,1\right)=1, \quad \operatorname{Max}\left(\mid T_{n}^{\prime} ;-1,1\right)=n^{2} .
\end{gathered}
$$

In diesen Bezeichnungen läßt sich der Satz des Herrn A. Markoff so aussprechen:

Für jedes Polynom

$$
f(x)=a_{0} x^{n}+a_{1} x^{n-1}+\ldots+a_{n-1} x+a_{n}
$$

besteht die Ungleichung

$$
\operatorname{Max}\left(f^{\prime} ; ;-1,1\right) \leqq n^{2}-\operatorname{Max}(|f| ;-1,1)
$$

und das Gleichheitszeichen gilt hier nur für Polynome $f(x)$ der Form const. $\left.T(x)^{3}\right)$.

Ein wichtiges Analogon zu diesem Satz verdankt man Herrn S. Bernstein $\left.{ }^{4}\right)$ :

Für jedes trigonometrische Polynom

$$
F(\varphi)=\frac{a_{0}}{2}+\sum_{\nu=1}^{n}\left(a_{v}, \cos \nu \varphi+b_{x} \sin \nu \varphi\right)
$$

3) Herr A. Mark off hat diesen Satz a. a. O. nur für reelle Koeffizienten $a_{v}$ bewiesen; daß er auch für komplexe $a$, gilt; hat zuerst Herr M. Riesz (I, S. 359) gezeigt.

4) "Sur l'oxdre de la meilleure approximation des fonctions continues par des polynomes de degré donné “, Mémoires publiés par la Classe des Sciences de l'Académie de Belgique, Bd. 4 (1912), S. 1-103 [S. 19-20]. Vgl. :auch L. Fejér, „Über konjugierte trigonometrische Reihen", Journal f. r. u. a. Math., Bd. 144 (1914), S. 48 bis 56 [S. 50, Fußnote], und M. Fekete, "Uber einen Satz des Herrn Serge Bernstein", ebenda Bd. 146 (1915), S. 88-94. 
besteht die Ungleichung

$$
\operatorname{Max}\left(\left|F^{\prime}(\varphi)\right| ; 0,2 \pi\right) \leqq n \cdot \operatorname{Max}(|F(\varphi)| ; 0,2 \pi) ;
$$

das Gleichheitszeichen gilt nur für Ausdrücke der Form const. $\sin n\left(\varphi-\varphi_{0}\right)$.

Für diesen Satz hat Herr M. Riesz (I, S. 356) einę besonders einfachen Beweis angegeben, der nur von der leicht abzuleitenden Identität

$$
F^{\prime}(\varphi)=\frac{1}{2 n} \sum_{\nu=1}^{2 n} F\left(\varphi+\varphi_{\nu}\right) \frac{(-1)^{p+1}}{1-\cos \varphi_{\nu}} \quad\left(\varphi_{n}=\frac{(2 v-1) \pi}{2 n}\right)
$$

Gebrauch macht. Herr Riesz hat auch gezeigt, daß der Markoffsche Satz sich aus dem Bernsteinschen verhältnismäßig leicht folgern läßt. Noch etwas direkter kann man folgendermaßen schließen.

Es genügt offenbar die Ungleichung

$$
\left|f^{\prime}(x)\right| \leqq M n^{2} \quad(M=\operatorname{Max}(\mid f ; ;-1,1))
$$

für $0 \leqq x \leqq 1$ zu beweisen. Für $0 \leqq x \leqq \cos \frac{\pi}{2 n}$ folgt dies unmittelbar, indem man $x=\cos \varphi$ setzt und den Bernsteinschen Satz auf das Kosinuspolynom $F(\varphi)=f(\cos \varphi$ ) anwendet (vgl. Riesz, I, S. 360). Denn aus.

$$
F^{\prime}(\varphi)=-\sin \varphi f^{\prime}(\cos \varphi), \quad\left|F^{\prime}(\varphi)\right| \leqq M n
$$

ergibt sich für $-1<x<1$

$$
\left|f^{\prime}(x)\right| \leqq \frac{M n}{\sqrt{1-x^{2}}}
$$

a.lso speziell für $0 \leqq x \leqq \cos \frac{\pi}{2 n}$

Um nun (2) auch für

$$
\left|f^{\prime}(x)\right| \leqq \frac{M n}{\sqrt{1-\cos ^{2} \frac{\pi}{2 n}}}=\frac{M n}{\sin \frac{\pi}{2 n}}<M n^{2}
$$

$$
\cos \frac{\pi}{2 n}<x \leqq 1
$$

zu beweisen, setze man $\iota_{\mu}=\cos \frac{\mu \pi}{n}$ und

$$
g(x)=\left(x^{2}-1\right) T_{n}^{\prime}(x)=2^{n-1} n \prod_{\mu=0}^{m}\left(x-c_{k}\right),
$$

so daß also

$$
g(\cos \varphi)=-n \sin \varphi \sin n \varphi
$$

wird. Nach der Lagrangeschen Interpolationsformel wird

also

$$
f(x)=\sum_{\mu=1}^{n} g_{\mu}(x) \frac{f\left(c_{\mu k}\right)}{g^{\prime}\left(\alpha_{k^{b}}\right)}, \quad\left(g_{a}(x)=\frac{g(x)}{x-\alpha_{\mu}}\right)
$$

$$
f^{\prime}(x)=\sum_{\mu=0}^{n} g_{\mu}^{\prime}(x) \frac{f\left(c_{\mu}\right)}{g^{\prime}\left(\alpha_{k l}\right)}
$$


Für $f(x)=T_{n}(x)$ geht diese Formel wegen

$$
\operatorname{sign} g^{\prime}\left(r_{\mu}\right)=(-1)^{\prime \prime}=T_{n}\left(c_{\mu r}\right)
$$

in

$$
T_{n}^{\prime}(x)=\sum_{n=0}^{n} \frac{g_{\mu}^{\prime}(x)}{g^{\prime}\left(x_{k}\right)}
$$

über. Ich behaupte nun, daß die $n+1$ Ausdrücke $g_{\mu}(x)$ im Intervall (4) nur positive Werte annehmen. Für $\mu=0$ ist das gewiß richtig, weil die Nullstellen von

$$
g_{0}^{\prime}(x)=\frac{d}{d x}\left(\begin{array}{c}
g(x) \\
x-1
\end{array}\right)
$$

links von $\cos \frac{\nu}{n}<\cos \frac{\pi}{2} n$ liegen und $\lim _{x \rightarrow \infty} g_{0}^{\prime}(x)=+\infty$ ist. Ist aber $n>0$, so wird in

$$
g_{\mu k}^{\prime}(x)=\frac{\left(x-\alpha_{\mu}\right) g^{\prime}(x)-g(x)}{\left(x-c_{u k}\right)^{2}}
$$

für $\cos \frac{\pi}{2 n}<x \leqq 1$

und $g^{\prime}(x)>0$, weil

$$
g(x) \leqq 0, \quad x-a_{n}>0
$$

$$
g^{\prime}(\cos \varphi)=\frac{1}{\sin \varphi}\left(n \cos \varphi \sin n \varphi^{\prime}+n^{2} \sin \varphi \cos n \varphi\right)
$$

für $0 \leqq \varphi<\frac{\pi}{2 n}$ positiv ist. Daher ist in der Tat $g_{\mu}^{\prime}(x)>0$ im Interxall (4) und folglich

$$
\left|f^{\prime \prime}(x)\right| \leqq \sum_{\mu=0}^{n} g_{\mu}^{\prime}(x) \frac{\left|f\left(\alpha_{\mu}\right)\right|}{\left|g^{\prime}\left(x_{k}\right)\right|} \leqq \sum_{\mu=0}^{n} g_{n}^{\prime}(x) \frac{M}{\left|g^{\prime}\left(\alpha_{\mu}\right)\right|}=M T_{n}^{\prime}(x) \leqq M n^{2} .
$$

Zugleich ergibt sich, daB in der nun bewiesenen Ungleichung (2) das Gleichheitszeichen nur dann stehen kann, wenn die $n+1$ Zahlen $f\left(c_{y}\right)$ sich von den entsprechenden Zahlen (6) nur um einen konstanten Faktor unterscheiden. Dann wird aber $f(x)=$ const. $T_{n}(x)$.

Beachtet man, daß die Funktionen $g_{j k}(x)$ nur reelle Nullstellen besitzen und daß $g_{\mu}(-x)=(-1)^{n} g_{n-\mu}(x)$ ist, so folgt aus dem über $g_{\mu}^{\prime}(x)$ Bewiesenen, daß auch die höheren Ableitungen von $g_{\mu}(x)$ außerhalb des Intervalls

$$
-\cos \frac{\pi}{2 n}<x<\cos \frac{\pi}{2 n}
$$

keinen Vorzeichenwechsel erleiden. Hieraus schließt man in derselben Weise wie vorhin für $f^{\prime}(x)$ im Intervall (4), daß für $k=1,2, \ldots, n$ außerhalb des Intervalls (8) die Ungleichung

$$
\left.\left|f^{(k)}(x)\right| \leqq M \cdot \mid T_{n}^{(k)}(x)\right\}
$$


besteht. Im Innern des Intervalls (8) ist, wie W. Markoff in seiner im Jahre 1892 erschienenen und neuerdings in den Mathematischen Annalen (Bd. 77 (1916), S. 213-258) wiederabgedruckten Abhandlung, ,Uber Polynome, die in einem gegebenen Intervalle möglichst wenig von Null abweichen" auf wesentlich komplizierterem. Wege bewiesen hat,

$$
\left|f^{(k)}(x)\right|<M \cdot T_{n}^{(k)}(1)=M \frac{n^{2}\left(n^{2}-1^{2}\right)\left(n^{2}-2^{2}\right) \ldots\left(n^{2}-(k-1)^{2}\right)}{1.3 .5 \ldots(2 k-1)} .
$$

Es wäre von Interesse, für diesen allgemeinen Satz einen ähnlich einfachen Beweis zu kennen wie für den Fall $k=1$.

\section{$\$ 2$.}

Präzisere Fassung des A. Markoffischen Satzes.

I. Ist $f(x)$ ein Polynom n-ten Grades, für das

ist, so ist 'stets

$$
\operatorname{Max}(f f ;-1,1)=M
$$

$$
\left|f^{\prime \prime}( \pm 1)\right| \leqq M n^{2}
$$

wobei das Gleichheitszeichen auszuschließen ist, wenn $f(x)$ nicht die Form const. $T_{n}(x)$ hat. Besitzt ferner, $\left\{f^{\prime}(x) \mid\right.$ an einer Stelle $\xi$ des Intervalls $-1 \leqq x \leqq 1$ ein (relatives) Maximum, so ist (für $n \geqq 3$ )

$$
\left|f^{\prime}(\xi)\right|<\frac{M n^{2}}{2}
$$

Der erste Teil dieses Satzes ist uns schon bekannt, der zweite Teil ergibt sich folgendermaßen. Haben die Zahlen $a_{p a}$ sowie die Ausdrücke $g(x)$ und $g_{\mu}(x)$ dieselbe Bedeutung wie in $\$ 1$, so liefert die Lagrangesche Interpolationsformel für alle Werte von $t$ und $x$

$$
f(t x)=\sum_{\mu=0}^{n} g_{k k}(t) \frac{f\left(x \alpha_{\mu k}\right)}{g^{\prime}\left(\alpha_{\mu k}\right)} .
$$

Differentiiert man nach $t$ und setzt dann $t=1$, so ergibt sich

$$
x f^{\prime}(x)=\sum_{\mu=0}^{n} g_{\mu}^{\prime}(1) \frac{f\left(x \alpha_{\mu}\right)}{g^{\prime}\left(\alpha_{\mu}\right)}=\sum_{\mu=0}^{n} A_{\mu} f\left(x \alpha_{\mu}\right) \text {. }
$$

Hierbei wird wegen (5)

$$
A_{0}=\frac{1}{g^{\prime}(1)}\left[\frac{d}{d t}\left(\frac{g(t)}{t-1}\right)\right]_{t=1}=\frac{g^{\prime \prime}(1)}{2 g^{\prime}(1)}=\frac{2 T_{n}^{\prime}(1)+4 T_{n}^{\prime \prime}(1)}{4 T_{n}^{\prime}(1)}=\frac{2 n^{3}+1}{6}
$$

und für $\mu>0$

$$
A_{\mu k}=\frac{\left(1-\alpha_{\mu}^{\prime}\right) g^{\prime}(1)-g(1)}{g^{\prime}\left(\alpha_{\mu}\right)\left(1-\alpha_{\mu}\right)^{2}}=\frac{g^{\prime}(1)}{g^{\prime}\left(\alpha_{\mu}\right)} \cdot \frac{1}{1-\alpha_{\mu}} .
$$

Mathematische Zeitschrift, IV. 
Die Formel ( 7 ) liefert nun

$g^{\prime}(1)=2 n^{2}, \quad g^{\prime}\left(c_{n}\right)=g^{\prime}(-1)=(-1)^{n} g^{\prime}(1), \quad g^{\prime}\left(a_{v}\right)=(-1)^{n} n^{2}$ $(v=1,2, \ldots, n-1)$.

Wir erhalten also die Identität

$$
x f^{\prime}(x)=\frac{2 n^{2}+1}{6} f(x)+\frac{(-1)^{n}}{2} f(-x)+\sum_{v=1}^{n-1} \frac{(-1)^{v}}{\sin ^{2} \frac{\nu \pi}{2 n}} f\left(x \cos \frac{p \pi}{n}\right),
$$

die für jedes Polynom $f^{\prime}(x)$ gilt, dessen Grund höchstens gleich $n$ ist. Daher ist auch

$$
x f^{\prime \prime}(x)=\frac{2 n^{2}+1}{6} f^{\prime}(x)+\frac{(-1)^{n}}{2} f^{\prime}(-x)+\sum_{n=1}^{n-1} \frac{(-1)^{\prime \prime}}{\sin ^{2} \frac{y^{\prime}}{2 n}} f^{\prime}\left(x \cos ^{\nu \pi} \frac{\pi}{n}\right) .
$$

Aus dieser Gleichung und der aus (10) durch Differentiation nach $x$ folgenden ergibt sich durch Addition

$$
f^{\prime}(x)+2 x f^{\prime \prime}(x)={ }^{2 n^{2}+1} f^{\prime}(x)+\sum_{j=1}^{n-1^{\mu \nu}}(-1)^{p+\ldots} \frac{1+\cos \frac{\nu \pi}{n}}{\sin ^{2} \frac{\nu \pi}{2 \cdot n}} f^{\prime}\left(x \cos \frac{\nu \pi}{n}\right)
$$

oder, was dasselbe ist,

$$
-\frac{n^{2}-1}{3} f^{\prime}(x)+x f^{\prime \prime}(x)=\sum_{n=1}^{n-1}(-1)^{\prime} \operatorname{ctg}^{2} \frac{{ }^{\prime} x}{2 n} f^{\prime}\left(x \cos \frac{y^{\prime} \pi}{n}\right) \text {. }
$$

Bedeutet nun $\bar{f}(x)$ dasjenige Polynom, das man aus $f(x)$ erhält, indem man alle Koeffizienten von $f(x)$ durch die konjugiert komplexen Werte ersetzt, so folgt aus (11) für reelle Werte von $x$

$-\frac{n^{2}-1}{3}\left|f^{\prime}(x)\right|^{2}+x \Re\left(f^{\prime \prime}(x) \bar{f}^{\prime} \cdot(x)\right)=\sum_{y=1}^{n-1}(-1)^{\prime \prime} \operatorname{ctg}^{2} \frac{\nu \pi}{2 n} \Re\left(\bar{f}^{\prime}(x) f^{\prime}\left(x \cos \frac{\nu \pi}{n}\right)\right)$.

Besitzt nun $\left|f^{\prime}(x)\right|$ an der Stelle $x=\xi$ ein Maximum, so gilt dasselbe für $\left|f^{\prime}(x)\right|^{2}=f^{\prime}(x) \bar{f}^{\prime}(x)$. und daher wird

also

$$
f^{\prime \prime}(\xi) \vec{f}^{\prime}(\xi)+f^{\prime}(\xi) \vec{f}^{\prime \prime}(\xi)=2 \Re\left(f^{\prime \prime}(\xi) \vec{f}^{\prime}(\xi)\right)=0,
$$

$$
-\left.\frac{n^{2}-1}{3} f^{\prime}(\xi)\right|^{2}=\sum_{v=1}^{n-1}(-1)^{\prime \prime} \operatorname{ctg} \frac{y \pi}{2 n} \Re\left(\bar{f}^{\prime}(\xi) f^{\prime}\left(\xi \cos \frac{v \pi}{n}\right)\right) \text {. }
$$

Hieraus folgt

$$
\frac{n^{2}-1}{3}\left|f^{\prime}(\xi)\right| \leqq \sum_{y=1}^{n-1} \operatorname{ctg}^{2} \frac{y^{\prime} \pi}{2}\left|f^{\prime}\left(\xi \cos \frac{y \pi}{n}\right)\right|
$$

Ist hierbei insbesondere $-1 \leqq \xi \leqq 1$, so wird wegen (3)

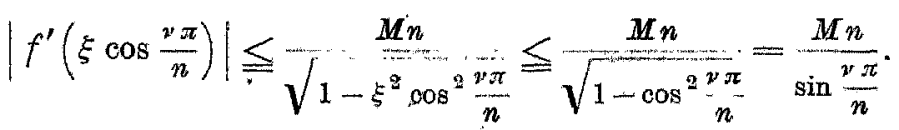


Ads (12) folgt daher

$$
f^{\prime}(\xi) \leqq \frac{3 M n}{2\left(n^{2}-1\right)} \sum_{n=1}^{n-1} \frac{\cos \frac{p \pi}{2 n}}{\left(\sin \frac{v^{n} \pi}{2 n}\right)^{3}}
$$

Da nun, wie man leicht zeigt, für $0<\varphi<\frac{\pi}{2}$

ist, so erhalten wir

$$
\varphi^{3} \cos \varphi<\sin ^{3} \varphi
$$

Für $n=3$ liefert dies

$$
f^{\prime}(\xi) \mid<\frac{12}{\pi^{\frac{3}{3}}} \cdot \frac{M n^{4}}{n^{2}-1} \sum_{v=1}^{n-1} \frac{1}{n^{3}}
$$

$$
f^{\prime}(\xi)<9 M \cdot{ }_{16 \pi^{3}}^{243}=9 M \cdot 0,486 \ldots
$$

und für $n \geqq 4$

$$
\left|f^{\prime}(\xi)\right|<M n^{2} \cdot \frac{12 n^{2}}{\pi^{3}\left(n^{2}-1\right)} \sum_{p=1}^{\infty} \frac{1}{p^{3}} \leqq M n^{2} \cdot \frac{12 \cdot 16}{15 x^{8}} \sum_{\nu=1}^{\infty} \frac{1}{y^{3}}=M n^{2} \cdot 0,496 \ldots
$$

Die zu beweisende Ungleichung (9) ist also in allen Fällen richtig.

Bedeutet $M^{\prime}$ das größte im Intervall $-1 \leqq x \leqq 1$ liegende relative Maximum von $\left|f^{\prime}(x)\right|$ and $m_{n}$ die obere Grenze der Zahlen $\frac{M^{\prime}}{\mathbb{M}^{\prime}}$ für alle Polynome des Grades $n$, so ergibt sich genaner

$$
\mu=\lim \sup _{n=\infty} m_{n} \leqq \frac{12}{\pi^{3}} \sum_{p^{\prime}=1}^{\infty} \frac{1}{p^{3}} \leq 0,465 \ldots
$$

Setzt man ferner insbesondere $f(x)=T_{n}(x)$, so wird $\left\{f^{\prime}(x)\right\}$ an den beiden Endpunkten des Intervalls $\cos \frac{2 \pi}{n} \leqq x \leqq \cos \frac{\pi}{n}$ gleich Null und besitzt demnach im Innern dieses Intervalls ein Maximum. Für jedes feste $\psi$ $z$ wischen 0 und $\pi$ ist daher

$$
m_{n} \geq \frac{1}{n^{2}}\left|T_{n}^{\prime}\left(\cos \frac{\pi+w}{n}\right)\right|=\frac{\sin \psi}{n \sin \frac{\pi+\psi}{n}}
$$

und folghich

$$
\mu \geqq \lim _{n=\infty} \frac{\sin \psi}{n \sin \frac{\pi+\psi}{n}}=\frac{\sin \psi}{\pi+\psi} .
$$

Das Maximum dieser Funktion von $\psi$ im Intervall $0 \leqq \psi \leqq \pi$ ist gleich $\cos \psi_{0}=0,217 \ldots$, wo $\psi_{0}$ die diesem Intervall angehörende Lösung der Gleichung tg $\psi=\pi+\psi$ bedeutet. Wir erhalten also

$$
0,217 \ldots \leqq \mu \leqq 0,465 \ldots
$$

Eine genauere Abschätzung dieser Konstanten $\mu$ dürfte nicht leicht sein. 
$\S 3$.

Über Polynome, die an einem Endpunkt des zu betrachtenden Intervalls versehwinden.

Daß wir uns bis jetzt auf die Betrachtung des Intervalls $(-1,1)$, beschränkt haben, ist natürlich ohne Bedeutung. Für ein beliebiges endliches Intervall $(a, b)$ lautet der A: Markoffsche Satz in unserer präziseren Fassung folgendermaßen: Ist $f(x)$ ein Polynom des Grades $n$ und

so wird stets

$$
\operatorname{Max}(|f| ; a ; b)=M
$$

$$
\left|f^{\prime}(a)\right| \leqq \frac{2 M n^{2}}{b-a}, \quad\left|f^{\prime}(b)\right| \leqq \frac{2 M n^{2}}{b-a}
$$

wobei das Gleichheitszeichen auszuschließen ist, wenn $f(x)$ nicht von der Form

$$
f(x)=\text { const. } T_{n}\left(\begin{array}{c}
2 x-a-b \\
b-a
\end{array}\right)
$$

ist. Besitzt ferner $\left|f^{\prime}(x)\right|$ an einer Stelle $\xi$ des Intervalls $(a, b)$ ein Maximum, so wird

$$
\left|f^{\prime}(\xi)\right|<\frac{M n^{2}}{b-a} .
$$

Auf Grund dieses Satzes läßt sich der A. Markoffsche Satz noch in folgender Weise ergänzen:

II. Ist $f(x)$ ein Polynom des Grades $n$, das an einem der beiden Endpunkte des Intervalls $a \leqq x \leqq b$ verschwindet, so ist stets

$$
\operatorname{Max}\left(\left|f^{\prime}\right| ; a, b\right) \leqq \frac{2 n^{2}}{b-a} \cos ^{2} \frac{\pi}{4 n} \cdot \operatorname{Max}(|f| ; a, b) .
$$

und es gibt auch Polynome $f(x)$ dieser Art, für die hier das Gleichheitszeichen gilt.

Es genügt offenbar, den Beweis für irgendein spezielles Intervall $(a, b)$ zu erbringen, und hier empfiehlt es sich $a=0, b=1$ zu wählen. Außerdem können wir uns auf den Fall

$$
f(0)=0, \quad \operatorname{Max}(|f| ; 0,1)=1
$$

beschränken und $n>1$ annehmen $\left.{ }^{5}\right)$. Wir haben nun zu zeigen, daß alsdann für $0 \leqq x \leqq 1$

$$
\left|f^{\prime}(x)\right| \leqq 2 n^{2} \cos ^{2} \frac{\pi}{4 n}
$$

5) Für $n=1$, kommt bier nur der Fall $f(x)=e^{i x x} x^{\prime}$ in Betracht, der auf $\left|f^{\prime}(x)\right|=1=2 \cdot 1^{2} \cos ^{2} \frac{\pi}{4}$ führt. 
wird. Ein spezielles Polynom $n$-ten Grades, das den Bedingungen (14) genügt, ist insbesondere

$$
U_{n}(x)=T_{n}\left(2 \cos \frac{\pi}{2 n} \cdot x-\cos \frac{\pi}{2 n}\right)=T_{n}\left(\left(1+\cos \frac{\pi}{2 n}\right) x-\cos \frac{\pi}{2 n}\right) .
$$

Dieser Ausdruck hat noch folgende Eigenschaften: An den $n$ Stellen

$$
\beta_{\nu}=\frac{\cos \frac{\nu \pi}{n}+\cos \frac{\pi}{2 n}}{1+\cos \frac{\pi}{2 n}} \quad(\nu=0,1, \ldots, n-1)
$$

des Intervalls $0 \therefore x \leqq 1$ wird $U_{n}(x)$ abwechselnd +1 und -1 , außerdem ist wegen (1)

$$
\begin{aligned}
& U_{n}^{\prime}(0)=2 \cos ^{2} \frac{\pi}{4 n} T_{n}^{\prime}\left(-\cos \frac{\pi}{2 n}\right)=(-1)^{n-1} n \operatorname{ctg} \frac{\pi}{4 n^{2}} \\
& U_{n}^{\prime}(1)=2 \cos ^{2} \frac{\pi}{4-n} T_{n}^{\prime}(1)=2 n^{2} \cos ^{2} \frac{\pi}{4 n} .
\end{aligned}
$$

Setzt man nun

$$
h(x)=x\left(x-\beta_{0}\right)\left(x-\beta_{1}\right), \ldots,\left(x-\beta_{n-1}\right)=x h_{1}(x),
$$

so folgt aus der Lagrangeschen Interpolationsformel

$$
f(x)=\sum_{\nu=0}^{n-1} \frac{h(x)}{x-\beta_{v}} \frac{f\left(\beta_{\nu}\right)}{h^{\prime}\left(\beta_{\nu}\right)}=x \sum_{v=0}^{n-1} \frac{h_{1}(x)}{x-\beta_{v}} \frac{f\left(\beta_{\nu}\right)}{h^{\prime}\left(\beta_{\nu}\right)} .
$$

Für $f(x)=U_{n}(x)$ läßt sich diese Gleichung wegen

$$
\operatorname{sign} h^{\prime}\left(\beta_{v}\right)=(-1)^{n}=U_{n}\left(\beta_{v}\right)
$$

insbesondere in der Form

$$
U_{n}(x)=\sum_{n=0}^{n-1} \frac{h(x)}{x-\beta_{n}} \frac{1}{\left|h^{\prime}\left(\beta_{p}\right)\right|}=x \sum_{n=1}^{n-1} \frac{h_{1}(x)}{x-\beta_{n}} \frac{1}{h^{i}\left(\beta_{n}\right) !}
$$

schreiben. Da nuin die Ableitungen der $n$ Funktionen $\frac{h(x)}{x-\beta_{y}}$ alle ihre Nullstellen im Intervall $0<x<1$ besitzen, so sind sie für $x \leqq 0$ und für $x \geqq 1$ von demselben Vorzeichen. Aus (17) und (18) folgt daher, daß für diese Werte von $x$

$$
\left.\left|f^{(m)}(x) \leqq \sum_{n=0}^{n-1}\right| \frac{d^{m}}{d x^{m}}\left(\frac{h(x)}{x-\beta_{r}}\right)\right|_{\left|h^{\prime}\left(\beta_{p}\right)\right|}=\left|U_{n}^{(m)}(x)\right\rangle \quad(m=0,1, \ldots, n)
$$

ist. Insbesondere wird

$$
\left|f^{\prime}(0)\right| \leqq n \operatorname{ctg} \frac{\pi}{4 n}, \quad\left|f^{\prime}(1)\right| \leqq 2 n^{2} \cos ^{2} \frac{\pi}{4 n},
$$

und hierbei ist die an zweiter Stelle stehende Sehrãnke die gxößere, denn dies besagt nur, da:B 


$$
2 \sin \frac{\pi}{4 n} \cos \frac{\pi}{4 n}=\sin \frac{\pi}{2 n}>\frac{2}{\pi} \cdot \frac{\pi}{2 n}=\frac{1}{n}
$$

ist. Um nun zu erkennen, daß die zu beweisende Ungleichung (15) auch für $0<x<1$ richtig ist, brauchen wir nur zu zeigen, daß für $0<\xi<1$

$$
f^{\prime}(\xi)<2 n^{2} \cos ^{2}{ }_{4 n}^{\pi}
$$

wird, sobald $\mid f^{\prime}(x)$ an der Stelle $x=\xi$ ein Maximum besitzt. Dies folgt aber aus (13), weil in unserem Falle

ist.

$$
\frac{M n^{2}}{b-a}=n^{2}=2 n^{2} \cos ^{2}{ }_{4}^{\pi}<2 n^{2} \cos ^{2}{ }_{4 n}^{\pi}
$$

Diese Betrachtung zeigt auch, dả für ein Polynom $f(x)$ des Grades $n>1$, das den Bedingungen (14) genügt, in der Ungleichung (15) das Gleichheitszeichen nur für $x=1$ stehen kann. Ferner tritt dies nur dann ein, wenn für $y=0,1, \ldots, n-1$

$$
f\left(\beta_{n}\right)=e^{i a t} \operatorname{sign} h^{\prime}\left(\beta_{n}\right)=e^{i a} U_{a s}\left(\beta_{n}\right)
$$

wird, d, h. wenn $f(x)$ von der Form $e^{i \alpha} U_{n}(x)$ ist.

\section{$\S 4$.}

\section{Fortsetzung.}

Die Formeln (17) und (18) gestatten uns, noch einen weiteren interessanten Satz abzuleiten:

\section{Für jedes Polynom}

$$
f(x)=a_{0} x^{n}+a_{1} x^{n-1}+\cdots+a_{n}
$$

das an der Stelle $x=0$ verschwindet und der Bedingung $\operatorname{Max}(|f| ; 0,1)=1$ genügt, ist

$$
a_{0} ! \leqq 2^{2 n-1}\left(\cos _{4 n}^{\pi}\right)^{2 n}
$$

und für $0 \leqq x \leqq 1$

$$
\frac{f(x)}{x} \leqq n \operatorname{ctg} \frac{\pi}{4 n} .
$$

In diesen beiden Ungleichungen sind die Gleichheitszeichen auszuschließen, wenn $f(x)$ nicht, von der Form $e^{i<} U_{n}(x)$ ist, und auch in diesem Fall gilt in (21) das Gleichheitszeichen nur für $\left.x=0^{8}\right)$.

Die Ungleichung (20) ergibt sich unmittelbar aus (19), indem man hierin $m=n$ setzt. Um (21) zu beweisen, setze man (vgl. Formel (16))

a) Unter $\frac{f(x)}{x}$ ist hierbei, wie überal im folgenden, die zugehörige ganze rationale Funktion zu verstehen 


$$
\beta=\beta_{n-1}=\frac{\cos \frac{\pi}{2 n}-\cos \frac{\pi}{n}}{1+\cos \frac{\pi}{2 n}}=\frac{\sin \frac{3 \pi}{4 n} \sin \frac{\pi}{4 n}}{\cos ^{2} \frac{\pi}{4 n}} .
$$

Dann wird für $\beta \leqq x \leqq 1$

$$
\left|\frac{f(x)}{x}\right| \leqq \frac{1}{\beta}=\frac{\cos \frac{\pi}{4 n}}{\sin \frac{3 \pi}{4 n}} \operatorname{ctg} \frac{\pi}{4 n}<\frac{1}{2} \frac{3 \pi}{4 n} \operatorname{ctg} \frac{\pi}{4 n}=2 n \operatorname{ctg} \frac{\pi}{4 n}
$$

so daß also (21) gewiß richtig ist. Für $x<\beta$ sind aber die in (17) auftretenden $n$ Ausdrücke $\frac{h_{1}(x)}{x-\beta}$, da $\beta$ die kleinste Nullstelle von $h_{1}(x)$ ist, von demselben Vorzeichen. Daher folgt aus (17) und (18)

$$
\left|\begin{array}{c}
f(x) \\
x
\end{array}\right| \leqq \sum_{v=0}^{n-1}\left|\begin{array}{c}
h_{1}(x) \\
x-\beta_{n} \mid h^{\prime}\left(\beta_{v}\right)
\end{array}\right|=\left|\frac{U_{n}(x)}{x}\right|,
$$

wobei das Gleichheitszeichen nur für $f(x)=e^{i *} U_{n}(x)$ gilt. Da nun

$$
\lim _{x \rightarrow 0}\left|U_{n}(x)=\right| U_{n}^{\prime}(0) \mid=n \operatorname{ctg} \frac{x}{4 x}
$$

ist, so haben wir nur noch zu zeigen, daß für $0 \ldots, x \ldots$

$$
U_{n}(x): x n \operatorname{ctg} \frac{\pi}{4 x}
$$

ist. Setzt man

$$
x=\frac{-\cos q+\cos 2 \pi}{2 n} \cdot \text {, also } U_{n}(x)=T_{n}(-\cos \varphi)=(-1)^{n} \cos n \varphi,
$$

so besagt (22) nur, daB für $\frac{\pi}{2 n}<\varphi<\frac{\pi}{n}$.

$$
F(\varphi)=n\left(\cos \frac{\pi}{2 n}-\cos \varphi\right)+\sin _{2 n}^{\pi} \cos n \varphi>0
$$

ist. Dies ist aber gewiß richtig, weil $F(\varphi)$ und $F^{\prime}(\varphi)$ für $\varphi=\frac{\pi}{2 n}$ verschwinden und

$$
F^{\prime \prime}(\varphi)=n \cos \varphi-n^{2} \sin \frac{\pi}{2 n} \cos n \varphi
$$

in dem Intervall $\frac{\pi}{2 n}<\varphi<\frac{\pi}{n}$ positiv ist.

Beachtet man, daß die Sätze II und III auch für Polynome mit komplexen Koeffizienten gelten, so ergibt sich allgemeiner:

IIT*. Es sei $z=z_{0}$ eine reelle oder komplexe $N$ ullstelle des Polynoms.

$$
f(z)=a_{0} z^{n}+a_{1} z^{n-1}+\ldots+a_{n-1} z+a_{n}
$$

und $z_{1}$ eine beliebige andere Stelle in der z-Ebene. Ist für alle Punkte z 
auf der geradlinigen Verbindungsstrecke der Punkte $z_{0}$ und $z_{1}$ der absolute Betrag von $f(z)$ höchstens gleich $M$, so ist für diese Werte von $z$

$$
\left|\frac{f(z)}{z-z_{0}}\right| \leqq \frac{M}{\left|z_{1}-z_{0}\right|} \cdot n \operatorname{ctg} \frac{\pi}{4 n}, \quad f^{\prime \prime}(z) \mid \leqq \frac{M}{\left|z_{1}-z_{0}\right|} \cdot 2 n^{2} \cos ^{2} \frac{\pi}{4 n} .
$$

F'erner ist

$$
\left|a_{0}\right| \leqq \frac{M}{\left|z_{1}-z_{0}\right|^{n}} \cdot 2^{2 n-1}\left(\cos \frac{\pi}{4 n}\right)^{2 n}
$$

Das Gleichheitszeichen ist in diesen Ungleichungen auszuschließen, wenn $f(z)$ nicht von der Form

ist.

$$
\text { const. } T_{n}\left(2 \cos ^{2} \frac{\pi}{4 n} \cdot \frac{z-z_{0}}{z_{1}-z_{0}}-\cos \frac{\pi}{2 n}\right)
$$

$\$ 5$.

Über Polynome, die an den beiden Endpunkten eines Intervalls verschwinden.

IV. Für jedes Polynom des Grades $n \geqq 2$

$$
f(x)=a_{0} x^{n}+a_{1} x^{n-1}+\ldots+a_{n-1} x+a_{n},
$$

das an den Stellen $x=0$ und $x=1$ verschwindet und der Bedingung $\operatorname{Max}(\lfloor f: 0 ; 1)=1$ genügt, ist

$$
\left.\left|a_{0}\right| \leqq 2^{2 n-1}\left(\cos \frac{\pi}{2 n}\right)^{n},{ }^{7}\right)
$$

ferner ist für $0 \leqq x \leqq 1$

$$
\left|f^{\prime}(x)\right| \leqq 2 n \operatorname{ctg} \frac{\pi}{2 n}, \quad\left|\frac{f(x)}{x(x-1)}\right| \leqq 2 n \operatorname{ctg} \frac{\pi}{2 n} .
$$

In diesen Ungleichungen ist das Gleichheitszeichen auszuschließen, wenn $f(x)$ nicht die Form $e^{i \alpha} V_{n}(x)$ hat; hierbei ist

$$
V_{n}(x)=T_{n}\left(\cos _{2}^{\pi} \cdot(2 x-1)\right)
$$

$z u$ setzen.

Der Beweis ergibtt sich ganz ähnlich wie in den Paragraphen 3 und 4. Setzen wir hier

$$
\gamma_{r}=\frac{\cos \frac{n \pi}{n}+\cos \frac{\pi}{2 n}}{2 \cos \frac{\pi}{2 n}} \quad(\nu=1,2, \ldots, n-1)
$$

7) Für Polynome mit reellen Koéffizienten findet sich diese Ungleiohung schon bei Tschebyschef, „Théorie des mécanismes connus sous le nom de parallélogammes", Werke Bd. I, S.111-143 'S.139!. 
und

$$
k(x)=x(x-1)\left(x-\gamma_{1}\right)\left(x-\gamma_{2}\right) \ldots\left(x-\gamma_{n-1}\right)=x(x-1) k_{1}(x),
$$

so wird

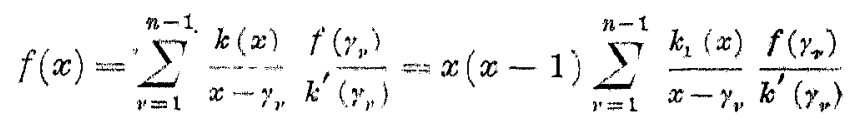

und speziell, weil

$$
\operatorname{sign} k^{\prime}\left(\gamma_{\nu}\right)=(-1)^{\prime \prime}=V_{n}\left(\gamma_{r}\right)
$$

ist,

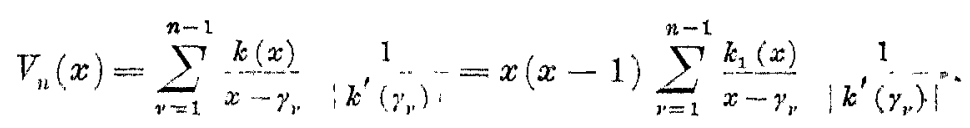

Hieraus folgt wieder für $x \leqq 0$ und $x \geqq 1$

$$
\left|f^{(m)}(x)\right| \leqq \sum_{v=1}^{n-1}\left|\frac{d^{m}}{d x^{m}}\left(\frac{k(x)}{x-y_{v}}\right)\right| \frac{1}{\left|k^{\prime}\left(\gamma_{v}^{\prime}\right)\right|}=\left|V_{n k}^{(m)}(x)\right| \quad(m=0,1, \ldots, n),
$$

was für $m=n$ insbesondere die Ungleiching (23) liefert. Da ferner $\nabla_{n}^{\prime}(0)=(-1)^{n-1} V_{n}^{\prime}(1)=(-1)^{n-1} \cdot 2 \cos \frac{\pi}{2 n} T_{n}^{\prime}\left(\cos \frac{\pi}{2 n}\right)=(-1)^{n-1} \cdot 2 n \operatorname{ctg} \frac{\pi}{2 n}$ ist, so erhalten wir

$$
\left|f^{\prime}(0)\right| \leqq 2 n \operatorname{ctg} \frac{\pi}{2 n}, \quad\left|f^{\prime}(1)\right| \leqq 2 n \operatorname{ctg}_{2 n}^{\pi} .
$$

Daß die erste der Ungleichungen (24) auch für $0<x<1$ gilt, folgt wieder, indem man beachtet, da $\beta$ an jeder Stelle $\xi$ dieses Intervalls, an $\operatorname{der}\left|f^{\prime}(x)\right|$ ein Maximum erreicht, wegen

$$
\left|f^{\prime}(\xi)\right|<n^{2}=n^{2} \cdot \frac{2}{2} \operatorname{ctg} \frac{\pi}{2 \cdot 2} \leqq n^{2} \cdot \frac{2}{n} \operatorname{ctg}_{2}^{2} \frac{\pi}{n}
$$

ist. Die hierbei benutzte Ungleiching für $\operatorname{ctg} \frac{\pi}{2 n}$ ist gewib richtig, da $\varphi$ ctg $\varphi$ im Intervall $0 \leqq \varphi \leqq \frac{\pi}{2}$ beständig abnimmt.

Beim Beweis der zweiten unter den Ungleichúngen (24) können wir uns offenbar auf das Intervall $0 \leqq x \leqq \frac{1}{2}$ beschränken und $n>2$ annehmen. Setzt man

so wird für $\gamma \leqq x \leqq \frac{1}{2}$

$$
\gamma=\gamma_{n-1}=\frac{1}{2}-\cos \frac{\pi}{n}, \frac{\pi}{2 \cos \frac{\pi}{2 n}},
$$

$$
\left|\frac{f(x)}{x(x-1)}\right| \leqq \frac{1}{\gamma(1-\gamma)} \frac{4 \cos \frac{\pi}{2 n}}{\sin \frac{3 \pi}{2 n}} \operatorname{ctg} \frac{\pi}{2 n}<\frac{4}{\frac{3}{3} \cdot \frac{3 \pi}{2 n}} \operatorname{ctg} \frac{\pi}{2 n}=\frac{2}{3} \cdot n \operatorname{ctg} \frac{\pi}{2 n} .
$$




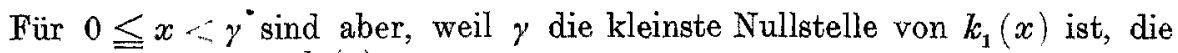
$n-1$ Ausdrücke $\frac{k_{1}(x)}{x-y_{2}}$ von demselben Vorzeichen. Aus (25) und (27) folgt daher

$$
\left|\frac{f(x)}{x(x-1)}\right| \leqq\left|\frac{V_{n}(x)}{x(x-1)}\right| .
$$

Wir haben also nur noch zu zeigen, daß für $0 \leqq x \leqq \gamma$

$$
\left|\frac{V_{n}(x)}{x(x-1)}\right| \leqq 2 n \operatorname{ctg}_{2 n}^{\pi}
$$

ist. Für $x=0$ ist hier das Gleichheitszeichen zu setzen, für $0<x<\gamma$ geht (28) durch die Substitution

$$
x=\frac{-\cos \varphi+\cos \frac{\pi}{2 n}}{2 \cos \frac{\pi}{2 n}^{\pi}} \quad\left(\frac{\pi}{2 n}<\varphi \cdot \frac{\pi}{n}\right)
$$

in die Ungleichung

$$
-\frac{4 \cos n \varphi \cos ^{2} \frac{\pi}{2} n}{\cos ^{2} \frac{\pi}{2 n}-\cos ^{2} q}<2 n \operatorname{ctg} \frac{\pi}{2 n}
$$

über, die nur besagt, daß

$$
G(\varphi)=\sin \frac{\pi}{n} \cos n \varphi+n\left(\cos ^{2} \pi_{n}^{\pi}-\cos ^{2} \varphi\right)
$$

im Intervall $\frac{\pi}{2 n}<\phi<\frac{\pi}{n}$ stets positiv ist. Dies ist aber gewiß richtig, denn für $n=3$ wird

$$
G\left(\phi^{\prime}\right)=4 \cos \frac{\pi}{6}\left(\cos ^{2} \frac{\pi}{6}-\cos ^{2} \varphi\right)\left(\cos \frac{\pi}{6}-\cos \varphi\right)
$$

und für $n>3$ ist $G\left(\frac{\pi}{2 n}\right)=G^{\prime}\left(\frac{\pi}{2 n}\right)=0$ und, solange $\varphi$ zwischen $\frac{\pi}{2 n}$ und $\frac{\pi}{n}$ bleibt,

$$
G^{\prime \prime}(\varphi)=-n^{2} \sin \frac{\pi}{n} \cos n \varphi+2 n \cos 2 \varphi>0 .
$$

Bei dieser Betrachtung ergibt sich zugleich, daß das Gleichheitszeichen in einer der Ungleichungèn (23) und (24) nur dann stehen kann, wenn die $n-1$ Ausdrücke $f\left(\gamma_{v}\right)$ sich von den entsprechenden Zahlen (26) nur um einen konstanten Faktor vom absoluten Betrage 1 unterscheiden, $d . h$. wenn $f(x)$ von der Form $e^{i \alpha} V_{n}(x)$ ist. Ist $n>2$, so gehen genauer auch in diesem speziellen Fall die Ungleichungen (24) nur für $x=0$ and $x=1$ in Gleichungen über.

Aus dem Satze IV folgt wieder allgemeiner:

IV** Sind $z_{0}$ und $z_{1}$ zwei reelle oder komplexe Wंurzeln der Gleichung

$$
f(z)=a_{0} z^{n}+a_{1} z^{n+1}+\ldots+a_{n-1} z+a_{n}=0
$$


und ist auf der geradlinigen Verbindungsstrecke dieser beiden Punkte $f(z) \mid \leqq M, \cdot$ so ist für alle Punkte auf dieser Strecke

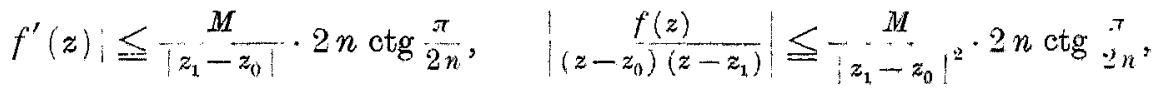
jerner ist

$$
a_{0} \mid \leqq \frac{2^{2 n-1}}{\left|z_{1}-z_{0}\right|^{n}}\left(\cos \frac{\pi}{2 n}\right)^{n} .
$$

In allen diesen Ungleichungen ist das Gleichheitszeichen auszuschließen, wenn $f(z)$ nicht von der Form.

ist.

$$
\text { const. } T_{n}\left(\frac{2 z-z_{0}-z_{1}}{z_{1}-z_{0}} \cdot \cos \frac{\pi}{2 n}\right)
$$

\section{$\$ 6$.}

Über Polynome, die im Mittelpunkt eines Intervalls versehwinden.

Der Vollständigkeit halber will ich noch auf einen weiteren Fall aufmerksam machen, der sich in ähnlicher Weise erledigen läßt.

V. Es sei $f(x)$ ein Polynom des Grades $n>2$, das an der Stelle $x=0$ verschwindet und der Bedingung $\operatorname{Max}(\mid f ;-1,1)=1$ genügt. Dann ist für $-1 \leqq x \leqq 1$

$$
\left|\frac{f(x)}{x}\right| \leqq m
$$

wobei $m$ gleich $n-1$ oder gleich $n$ zu setzen ist, je nachdem $n$ gerade oder ungerade ist. Nur dann, wenn $n$ ungerade und $f(x)=e^{i n} T_{n}(x)$ ist, kann in (29) das Gleichheitszeichen stehen, und zwar allein für $x=0$. Aber auch für ein gerades $n$ kann in (29), solange $f(x)$ keiner weiteren. Bedingung unterworfen wird, die obere Schranke $m$ durch keine kleinere Zahl ersetzt werden.

Setzt man $\iota_{\mu}=\cos _{m}^{\mu \pi}, \quad l(x)=x\left(x^{2}-1\right) T_{m}^{\prime}(x)=2^{n-1} n x \prod_{m=0}^{m}\left(x-\iota_{k t}\right)=x l_{1}(x)$, so wird sowohl für gerade als auch für ungerade Werte von $n$

$$
f(x)=\sum_{\mu=0}^{m} \frac{l(x)}{x-a_{y r}} \cdot \frac{f\left(c_{t}\right)}{l^{\prime}\left(c_{\mu}\right)} .
$$

Diese Formel gilt insbesondere auch für ' $f(x)=T_{m}(x)$, weil $T_{m}(x)$ für ungerades $m$ an der Stelle $x=0$ verschwindet. Nun ist aber, wenn $\sigma_{k *}$ das Vorzeichen von $a_{w}$ bedeutet,

$$
T_{m}\left(c_{\mu}\right)=(-1)^{\mu}=\operatorname{sign} l_{1}^{\prime}\left(c_{\mu}\right)=\sigma_{k^{\prime}} \cdot \operatorname{sign} l^{\prime}\left(c_{m}\right) .
$$


Aus (30) folgt daher.

Setzt man

$$
T_{n k}(x)=-\cdots \sum_{n=0}^{m} \frac{l(x)}{x-\alpha_{\mu}} \cdot \frac{\sigma^{\prime \prime}}{\left|l^{\prime}\left(\alpha_{\mu}\right)\right|} .
$$

$$
r=c_{\frac{m-1}{3}}=\cos \frac{(m-1)}{2 m} \pi \sin \frac{\pi}{2 m}
$$

so sind $-a$ und $a$ die absolut kleinsten unter den Zahlen $a_{\mu}$. Hieraus folgt leicht, daß für $-a<x<0$ und für $0<x<\ll$

$$
\operatorname{sign} \frac{l(x)}{x=\alpha_{\mu \prime}}=(-1)^{\frac{m-1}{3}} \sigma_{\mu} \operatorname{sign} x
$$

ist. Für diese Werte von $x$ (und also auch für $x=0$ ) ergibt sich daher aus (30) and (31)

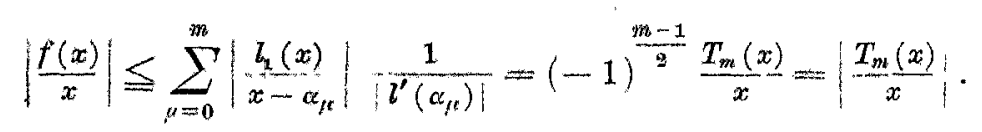

Setzt man nun $x=\sin \varphi$, so wird $T_{m}(x)=\cos m\left(\frac{\pi}{2}-\varphi\right)=(-1)^{\frac{m-1}{2}} \sin m \varphi$ und wir erhalten für $-\epsilon<x<k$, d. h. fürr $-\frac{\pi}{2 m}<\phi<\frac{\pi}{2 m}$

$$
|f(x)| \leqq\left|\frac{\sin m \varphi}{\sin \varphi}\right| \leqq m
$$

Ist ferner $u \leqq x^{i} \leqq 1$, so wird

$$
\left|\frac{f(x)}{x}\right| \leqq \frac{1}{\alpha}=\frac{1}{\sin \frac{\pi}{2 m}}<\frac{1}{\frac{2}{\pi} \cdot \frac{\pi}{2 m}}=m .
$$

Damit ist die Ungleichung (29) für das ganze Intervall $-1 \leqq x \leqq 1$ bewiesen. Das Gleichheitszeichen kommt hierbei nur danh in Betracht, wenn es in (:32) und (33) überall gilt. Dann muß aber $x=0$ sein und außerdem müssen sich die $m+1$ Zahlen $f\left(c_{\mu}^{*}\right)$ von den entsprechenden Zahlen $T_{m}\left(c_{\mu}\right)$ nur um einen Falktor der Form $e^{i \alpha}$ unterscheiden. Da hierzu noch $f(0)=T_{m}(0)=0$ hinzukommt, so muß der Grad $n$ von $f(x)$ gleich $m$ und $f(x)=e^{i \alpha} T_{m}(x)$ sein. Für ein gerades $n>2$ ist also in (29) das Gleichheitszeichen auszuschließen ${ }^{8}$ ). Setzt .man aber, wenn $\varepsilon$ irgendeine positive Zahl bedeutet,

so wird

$$
f(x)=\frac{1+\delta x}{1+\varepsilon} T_{m}(x)
$$

$$
\lim _{x=0}\left|\frac{f(x)}{x}\right|=\frac{m}{1+\varepsilon}
$$

-) Die Ungleichnng (29) ist auch für $n=2$ richtig, hierbei kann aber, wenn $x= \pm 1$ ist, das Gleichheitszeichen gelten. 
und da dieser Ausdruck dem Werte $m$ beliebig nahe gebracht werden kann, so läßt sich in (29) auch für ein gerades $n$ die obere Schranke $m$ durch keine kleinere Zahl ersetzen.

In ähnlicher Weise wie in den früheren Fällen können wir aus $V$ allgemeiner schließen:

$V^{*}$. Es sei $f(z)$ ein Polynom $n$-ten Grades, das an der stelle $z_{0}$ verschwindet. Sind $z_{1}$ und $z_{3}$ zwei in bezug auf $z_{0}$ symmetrisch gelegene Punkte, und ist auf der geradlinigen Strecke, die $z_{1}$ und $z_{2}$ verbindet, $|f(z)| \leqq M$, so ist für diese Werte von $z$

$$
\left|\frac{f(z)}{z-z_{0}}\right| \leqq M(n-1) \quad \text { oder } \quad\left|\frac{f(z)}{z-z_{0}}\right| \leqq M n,
$$

je nachdem $n$ gerade oder ungerade ist. Für $n>2$ gilt hierbei das Gleichheitszeichen nur dann, wenn $n$ ungerade, $z=z_{0}$ und $f(z)$ von der Form const. $T_{n}\left(\frac{z-z_{0}}{z_{1}-z_{0}}\right) i s t$.

(Eingegangen am 11. Januar 1919.) 